\title{
A STRINGENT LIMIT ON THE AMPLITUDE OF ALFVÉNIC PERTURBATIONS IN HIGH-BETA LOW-COLLISIONALITY PLASMAS
}

\author{
J. SQuire ${ }^{1,2}$, E. Quataert ${ }^{3}$, and A. A. Schekochinin ${ }^{4,5}$ \\ ${ }^{1}$ Theoretical Astrophysics, California Institute of Technology, 350-17, Pasadena, CA 91125, USA; jsquire@ caltech.edu \\ ${ }^{2}$ Walter Burke Institute for Theoretical Physics, Pasadena, CA 91125, USA \\ ${ }^{3}$ Astronomy Department and Theoretical Astrophysics Center, University of California, Berkeley, CA 94720, USA \\ ${ }^{4}$ The Rudolf Peierls Centre for Theoretical Physics, University of Oxford, 1 Keble Road, Oxford OX1 3NP, UK \\ ${ }^{5}$ Merton College, Oxford OX1 4JD, UK \\ Received 2016 August 16; revised 2016 September 15; accepted 2016 September 16; published 2016 October 12
}

\begin{abstract}
It is shown that low-collisionality plasmas cannot support linearly polarized shear-Alfvén fluctuations above a critical amplitude $\delta B_{\perp} / B_{0} \sim \beta^{-1 / 2}$, where $\beta$ is the ratio of thermal to magnetic pressure. Above this cutoff, a developing fluctuation will generate a pressure anisotropy that is sufficient to destabilize itself through the parallel firehose instability. This causes the wave frequency to approach zero, interrupting the fluctuation before any oscillation. The magnetic field lines rapidly relax into a sequence of angular zig-zag structures. Such a restrictive bound on shear-Alfvén-wave amplitudes has far-reaching implications for the physics of magnetized turbulence in the high- $\beta$ conditions prevalent in many astrophysical plasmas, as well as for the solar wind at $\sim 1$ au where $\beta \gtrsim 1$.
\end{abstract}

Key words: galaxies: clusters: intracluster medium - magnetic fields - plasmas - turbulence - waves

\section{INTRODUCTION}

Shear-Alfvén waves are perhaps the most fundamental of all oscillations in a magnetized plasma (Alfvén 1942). Their existence provides a key distinction between neutral and magnetized fluids, and they play a central theoretical role in most sub-disciplines of plasma physics, including magnetized turbulence (Goldreich \& Sridhar 1995; Ng \& Bhattacharjee 1996), the solar wind (Ofman 2010; Bruno \& Carbone 2013), the solar corona (Marsch 2006), and magnetic fusion (Heidbrink 2008). This general applicability has led to intense study of their basic properties (Cramer 2011). This researchwhich includes studies ranging from kinetic physics and damping mechanisms (Foote \& Kulsrud 1979), to nonlinear instabilities (Hamabata 1993; Medvedev et al. 1997) and the effects of inhomogeneity (Velli 1993) — has in turn been vital for the formulation of more applied theories. Interestingly, the low-frequency shear-Alfvén wave specifically has emerged relatively unscathed from this onslaught of theoretical inquiry (but see Del Zanna et al. 2001; Cramer 2011; Bruno \& Carbone 2013 and references therein), apparently being much less affected by kinetic damping mechanisms and other nonideal effects than its fast and slow wave cousins (Foote \& Kulsrud 1979; Schekochihin et al. 2007).

In this Letter, we discuss a dramatic departure from this behavior, showing that a high-beta collisionless plasma cannot support linearly polarized shear-Alfvén (SA) fluctuations above the critical amplitude,

$$
\left(\delta B_{\perp} / B_{0}\right)_{\max } \sim \beta^{-1 / 2},
$$

where $\beta \equiv 8 \pi p_{0} / B_{0}^{2}$ is the ratio of thermal pressure to magnetic pressure. This upper bound is independent of the spatial scale of the perturbation (as long as it is above the ion Larmor radius), and a similar restriction also holds in the weakly collisional Braginskii limit (Braginskii 1965). For fluctuations with $\delta B_{\perp} / B_{0} \gtrsim \beta^{-1 / 2}$, the magnetic field rapidly forms a sequence of zig-zags—straight field-line segments joined by sharp corners-maintaining this configuration with the magnetic energy far in excess of the kinetic energy.

What is the cause of such dramatic nonlinear behavior, even in regimes $\left(\delta B_{\perp} / B_{0} \ll 1\right.$ for $\left.\beta \gg 1\right)$ where linear physics might appear to be applicable? In a magnetized plasma in which the ion gyro-frequency $\Omega_{c}$ is much larger than the collision frequency $\nu_{c}$, a decreasing (in time) magnetic field leads-due to conservation of particle magnetic moment $\mu=m v_{\perp}^{2} / 2 B$ - to a decreasing pressure perpendicular to the magnetic field $\left(p_{\perp}\right)$, while the parallel pressure $\left(p_{\|}\right)$increases. This anisotropy, $\Delta p \equiv p_{\perp}-p_{\|}<0$, neutralizes the restoring effects of magnetic tension, destabilizing the SA wave if $\Delta p<-B^{2} / 4 \pi$. This well-studied instability is known as the parallel firehose (Rosenbluth 1956; Chandrasekhar et al. 1958; Parker 1958; Schekochihin et al. 2010). Now consider the ensuing dynamics if we start with $\Delta p=0$, but with a field that, in the process of decreasing due to the Lorentz force, generates a pressure anisotropy that would be sufficient to destabilize itself. This is a nonlinear effect not captured in linear models of SA waves. As $\Delta p$ approaches the firehose limit, the magnetic tension disappears and the Alfvén frequency approaches zero, interrupting the development of the wave. As shown below, because the wave perturbs the field magnitude by $\delta B_{\perp}^{2}$, an amplitude $\delta B_{\perp} / B_{0} \gtrsim \beta^{-1 / 2}$ is sufficient to generate such a $\Delta p$ in a collisionless plasma. As the field decrease is interrupted at the firehose stability boundary, the plasma self-organizes to prevent further changes in field strength, leading to the development of piecewise-straight (and therefore, tension-less) field-line structures.

This Letter explores the physics of this stringent amplitude limit, starting with simple analytic considerations. We then numerically examine the nonlinear behavior of fluctuations with amplitudes that exceed the limit and conclude with a discussion of possible implications for astrophysical turbulence and the solar wind. We focus primarily on the fate of an isolated $B_{\perp}$ perturbation-i.e., a linearly polarized standing wave-because this case is the simplest physically. Both the amplitude limit itself, and the plasma dynamics as the system 
approaches the firehose limit, are similar for traveling waves and for an initial velocity perturbation. Circularly polarized perturbations are, however, unaffected.

\section{THEORY}

On spatiotemporal scales larger than those relating to particle gyromotion, the particle distribution function is approximately gyrotropic. The magnetic field and first three moments of the kinetic equation then satisfy (Kulsrud et al. 1983; Schekochihin et al. 2010)

$$
\begin{gathered}
\partial_{t} \rho+\nabla \cdot(\rho \boldsymbol{u})=0, \\
\rho\left(\partial_{t} \boldsymbol{u}+\boldsymbol{u} \cdot \nabla \boldsymbol{u}\right)=-\nabla\left(p_{\perp}+\frac{B^{2}}{8 \pi}\right)+\nabla \cdot\left[\hat{\boldsymbol{b}} \hat{\boldsymbol{b}}\left(\Delta p+\frac{B^{2}}{4 \pi}\right)\right], \\
\partial_{t} \boldsymbol{B}=\nabla \times(\boldsymbol{u} \times \boldsymbol{B}), \\
\partial_{t} p_{\perp}+\nabla \cdot\left(p_{\perp} \boldsymbol{u}\right)+p_{\perp} \nabla \cdot \boldsymbol{u}+\nabla \cdot\left(q_{\perp} \hat{\boldsymbol{b}}\right)+q_{\perp} \nabla \cdot \hat{\boldsymbol{b}} \\
=p_{\perp} \hat{\boldsymbol{b}} \cdot(\hat{\boldsymbol{b}} \cdot \nabla \boldsymbol{u})-\nu_{c} \Delta p, \\
\partial_{t} p_{\|}+\nabla \cdot\left(p_{\|} \boldsymbol{u}\right)+\nabla \cdot\left(q_{\|} \hat{\boldsymbol{b}}\right)-2 q_{\perp} \nabla \cdot \hat{\boldsymbol{b}} \\
=-2 p_{\|} \hat{\boldsymbol{b}} \cdot(\hat{\boldsymbol{b}} \cdot \nabla \boldsymbol{u})+2 \nu_{c} \Delta p,
\end{gathered}
$$

where Gauss units are used, $\boldsymbol{u}$ and $\boldsymbol{B}$ are the plasma flow velocity and magnetic field, $\rho$ is the mass density, $B \equiv|\boldsymbol{B}|$ and $\hat{\boldsymbol{b}}=\boldsymbol{B} / B$ denote the field strength and direction, and $q_{\perp}$ and $q_{\|}$ are heat fluxes along $\hat{\boldsymbol{b}}$ associated with the perpendicular and parallel thermal energies, respectively. We also define $\Delta \equiv \Delta p / p_{0}$ with $p_{0}=2 p_{\perp} / 3+p_{\|} / 3$ (note $\Delta p \ll p_{0}$ for $\beta \gg 1)$ and $v_{A}=B_{0} / \sqrt{4 \pi \rho}$. While Equations (2)-(6) will be solved numerically below (Figure 1), in this section we make various approximations to derive analytically the amplitude limits and simplified wave equations. We consider two approximations for $\Delta p-$ one collisionless $\left(\nu_{c}=0\right)$, the other weakly collisional (Braginskii; $\Omega_{c} \gg \nu_{c} \gg|\nabla \boldsymbol{u}|$ )-neglecting compressibility in both cases (valid for $\beta \gg 1, \delta B_{\perp} / B_{0} \ll 1$ ).

When $d B / d t<0$, the terms $\hat{\boldsymbol{b}} \cdot(\hat{\boldsymbol{b}} \cdot \nabla \boldsymbol{u}) \approx B^{-1} d B / d t$ in Equations (5)-(6) locally force $\Delta=\Delta p / p_{0}<0$. Let us first consider collisionless $\left(\nu_{c}=0\right)$ evolution of $\Delta$, which is strongly influenced by heat fluxes for $\beta \gtrsim 1$. As a simple prescription for $q_{\perp, \|}$, we use a successful Landau fluid (LF) closure (Snyder et al. 1997), which (with $\Delta \ll 1$ ) posits

$$
q_{\|} \approx-2 \rho \sqrt{\frac{2}{\pi} \frac{p_{\|}}{\rho}} \frac{k_{\|}}{\mid k_{\|}}\left(\frac{p_{\|}}{\rho}\right), q_{\perp} \approx-\rho \sqrt{\frac{2}{\pi} \frac{p_{\|}}{\rho}} \frac{k_{\|}}{\left|k_{\|}\right|}\left(\frac{p_{\perp}}{\rho}\right) .
$$

Further assuming $\hat{\boldsymbol{b}} \cdot \nabla q_{\perp, \|} \gg q_{\perp, \|} \nabla \cdot \hat{\boldsymbol{b}}$ (valid at $\delta B_{\perp} / B_{0} \ll 1$ ) and using $p_{\|} / \rho \approx p_{0} / \rho=c_{s}^{2}$, one obtains $\nabla \cdot\left(q_{\perp} \hat{\boldsymbol{b}}\right)+q_{\perp} \nabla$. $\hat{\boldsymbol{b}} \sim-\rho c_{s} \mid k_{\|}\left(p_{\perp} / \rho\right)$ in the $p_{\perp}$ Equation (5) (similarly for $p_{\|}$, Equation (6)). This term, which models Landau damping of temperature perturbations, suppresses spatial variation in $p_{\perp, \|}$ over the particle crossing time $\tau_{\text {damp }} \sim\left(\left|k_{\|}\right| c_{s}\right)^{-1}$. Thus, if $\tau_{\text {damp }} \ll|\nabla \boldsymbol{u}|^{-1}$, the $k_{\|} \neq 0$ part of $\Delta$ is suppressed by $\sim v_{A} / c_{s} \sim \beta^{-1 / 2}$ compared to its mean, and a simple model is that $q_{\perp, \|}$ act to spatially average the $\Delta p$ driving, or

$$
\begin{aligned}
\Delta & =3 \int\langle\hat{\boldsymbol{b}} \cdot(\hat{\boldsymbol{b}} \cdot \nabla \boldsymbol{u})\rangle d t\left[1+\mathcal{O}\left(\beta^{-1 / 2}\right)(\boldsymbol{x})\right] \\
& \approx 3\left\langle\ln \frac{B(t)}{B(0)}\right\rangle .
\end{aligned}
$$

Now consider the Braginskii limit, where collisions dominate $\left(\nu_{c} \gg \nabla \boldsymbol{u}\right)$. Equations (5) and (6) then give

$$
\Delta \approx \nu_{c}^{-1} \hat{\boldsymbol{b}} \cdot(\hat{\boldsymbol{b}} \cdot \nabla \boldsymbol{u}),
$$

neglecting $q_{\perp, \|}$ for simplicity (valid for $\delta p_{\perp, \|} / p_{\perp, \|} \ll|\boldsymbol{u}| / c_{s}$ ).

Furnished with approximations for $\Delta p$ (Equations (8) or (9)), we now examine SA fluctuation dynamics. Consider a background field $B_{0} \hat{z}$, with perturbations perpendicular to $\hat{z}$ and the wavevector $\boldsymbol{k}=k_{\|} \hat{\boldsymbol{z}}+\boldsymbol{k}_{\perp}$. Since SA waves are unmodified by $\boldsymbol{k}_{\perp} \neq 0$ (the envelope is simply modulated in the perpendicular direction) and we analyze linear polarizations, we take $x$-directed perturbations that depend only on $z$ and $t ; \quad \boldsymbol{B}=B_{0} \hat{z}+\delta B_{\perp}(z, t) \hat{\boldsymbol{x}}, \quad \boldsymbol{u}=u_{\perp}(z, t) \hat{\boldsymbol{x}}$. Neglecting compressibility, the field perturbation $\delta b=\delta B_{\perp} / B_{0}$ satisfies

$$
\frac{\partial^{2}}{\partial t^{2}} \delta b=v_{A}^{2}\left[\frac{\partial^{2}}{\partial z^{2}} \delta b+\frac{\beta}{2} \frac{\partial^{2}}{\partial z^{2}}\left(\frac{\delta b}{1+\delta b^{2}} \Delta(z)\right)\right] .
$$

Equation (10) illustrates that in the absence of a background $\Delta$ linear long-wavelength SA, fluctuations are unmodified by kinetic effects, while the parallel firehose occurs because the coefficient of $\partial_{z}^{2}(\delta b)$ is negative for $\beta \Delta / 2<-1$.

Combining Equations (8) and (10), we see that if a collisionless wave evolves such that $3\langle\ln [B(t) / B(0)]\rangle=-2 / \beta$, its restoring force disappears. As we now explain, although the amplitude limit in each case is the same, standing and traveling waves differ in why a decrease in $\langle B(t)\rangle$ occurs. In a standing wave starting from a magnetic perturbation, $\langle B(t)\rangle$ simply decreases as the wave evolves. Thus, if $-3\left\langle\ln \left[B_{0} / B(0)\right]\right\rangle \approx 3 \delta b(0)^{2} / 4>2 / \beta$ (assuming $\left.\delta b(0) \sim \delta b_{0} \sin \left(k_{\|} z\right) \ll 1\right)$, an interruption occurs before a quarter period, implying the maximum wave amplitude is

$$
\left(\frac{\delta B_{\perp}}{B_{0}}\right)_{\max } \approx \sqrt{\frac{8}{3}} \beta^{-1 / 2} .
$$

This limit is matched nearly perfectly by numerical LF solutions (see Figure 2). A standing wave with an initial velocity perturbation also satisfies the limit (11) and is addressed in more detail below. For traveling waves, a crucial role is played by the spatially dependent $\mathcal{O}\left(\beta^{-1 / 2}\right)$ part of $\Delta$, which we neglected for convenience in deriving Equation (11). This role is to decrease $\langle B(t)\rangle$ by damping the wave nonlinearly. This "pressure-anisotropy damping" is related to correlations between $B^{-1} d B / d t$ and $\Delta p$, which cause a contribution to the rate of change of thermal energy of the form $\sim \int d x \Delta p B^{-1} d B / d t$ (see Equations (4)-(6)). Because this is positive for a traveling wave, the wave heats the plasma and damps at the rate $\sim \omega_{A} \delta b^{2} \beta^{1 / 2}$ (where $\omega_{A}=k_{\|} v_{A}$ ). Without any mechanism to isotropize the pressure, the decrease in $\langle B(t)\rangle$ causes $\langle\Delta\rangle$ to decrease as $\langle\Delta\rangle=3\langle\ln [B(t) / B(0)]\rangle$ (Equation (8)), which slows the wave (see Figure 1(c)) before stopping it completely if $\langle\Delta\rangle=-2 / \beta$. The maximum amplitude of a traveling wave is thus also given by Equation (11), although the time to approach the limit is 
increased compared to the standing wave because of the time required for the wave to damp nonlinearly.

A similar estimate of the amplitude limit with the Braginskii closure (9), using $\partial_{t}(\delta b) \sim \omega_{A} \delta b$, yields

$$
\frac{\beta}{2} \frac{\omega_{A}}{\nu_{c}} \delta b(0)^{3} \lesssim \delta b(0) \quad \Longrightarrow \quad \delta b(0)_{\max } \sim \sqrt{\frac{\nu_{c}}{\omega_{A}}} \beta^{-1 / 2}
$$

Since $\nu_{c} / \omega_{A} \gg 1$ for the validity of Braginskii's approximation, this condition is less stringent than Equation (11); note also that it depends on $k_{\|}\left(\right.$via $\left.\omega_{A}\right)$ unlike the collisionless case. In the Braginskii limit, traveling waves are again strongly damped (at the rate $\left.\sim\left(\omega_{A} / \nu_{c}\right) \delta b^{2} \beta \omega_{A}\right)$ due to the spatial correlation of $\Delta$ and $B^{-1} d B / d t$.

\section{NONLINEAR EVOLUTION AND NUMERICAL RESULTS}

The results above naturally invite the question: what happens to fluctuations above the critical amplitude? Here we illustrate, through numerical solutions and simple arguments, the remarkable tendency of collisionless plasmas to minimize the variation in $B^{2}$ (Kunz et al. 2014; S. Melville \& A. A. Schekochihin 2016, in preparation; Melville et al. 2016; Rincon et al. 2016). As a result, an initially sinusoidal $\delta b$ relaxes into a square wave, corresponding to zig-zags in the field lines. This peculiar behavior also emerges from Equations (8) and (10), despite their simplicity, illustrating the effect's simple physical origins. Solutions using Braginskii MHD differ in appearance and damp to fluctuations with $\delta b<(\delta b)_{\max }$.

We solve Equations (2)-(6) with the LF closure, using a dealiased pseudo-spectral method and hyperviscously damping all variables to remove energy just above the grid scale. Our only further approximation is the identification of $1 /\left|k_{\|}\right|$in Equation (7) with $1 /\left|k_{z}\right|$ (valid for $\delta b \ll 1$ ). The full equations solved are Equations (35)-(44) of Sharma et al. (2006) (except we use $1 /\left|k_{z}\right|$ in Equation (7), not their $k_{L}$ ). We do not artificially limit the pressure anisotropy to the firehose limit, as is common in previous turbulence studies (Sharma et al. 2006; Santos-Lima et al. 2014). This is because the parallel firehose instability - the cause of the effect-is in fact captured by the fluid model. In addition, since finite Larmor radius effects (FLRs) are not contained in this LF model, all scales in the simulation are larger than the gyroradius. ${ }^{6}$

The evolution of a sinusoidal SA perturbation is shown in Figure 1, starting with a perturbation in either (a) $\boldsymbol{B}$, (b) $\boldsymbol{u}$, or (c) a traveling wave. For comparison, we show solutions of the nonlinear wave Equation (10) in panels (d)-(e). In panels (b)(c), $\Delta$ is limited at the mirror threshold $\Delta=1 / \beta$, since $d B / d t>0$ in some regions (see the discussion below). We see from Figures 1(a)-(d) that collisionless waves-both standing and traveling - generically relax to a stable sequence of nearperfect stair steps. The spatial scale of the jumps is set by the numerics, so would likely be determined by FLR effects in reality. The basic origin of such structures can be understood by observing that if $\langle\Delta\rangle=-2 / \beta$ in Equation (10), $\partial_{z}^{2}(\delta b)+(\langle\Delta\rangle \beta / 2) \partial_{z}^{2}(\delta b)=0$. Neglecting residual spatial variation in $\Delta$ (this decreases after wave interruption because $B^{-1} d B / d t$ decreases), the remainder on the right-hand side

\footnotetext{
6 Firehose fluctuations are damped due to hyperviscosity, which thus determines the scale of the fastest growing firehose modes.
}

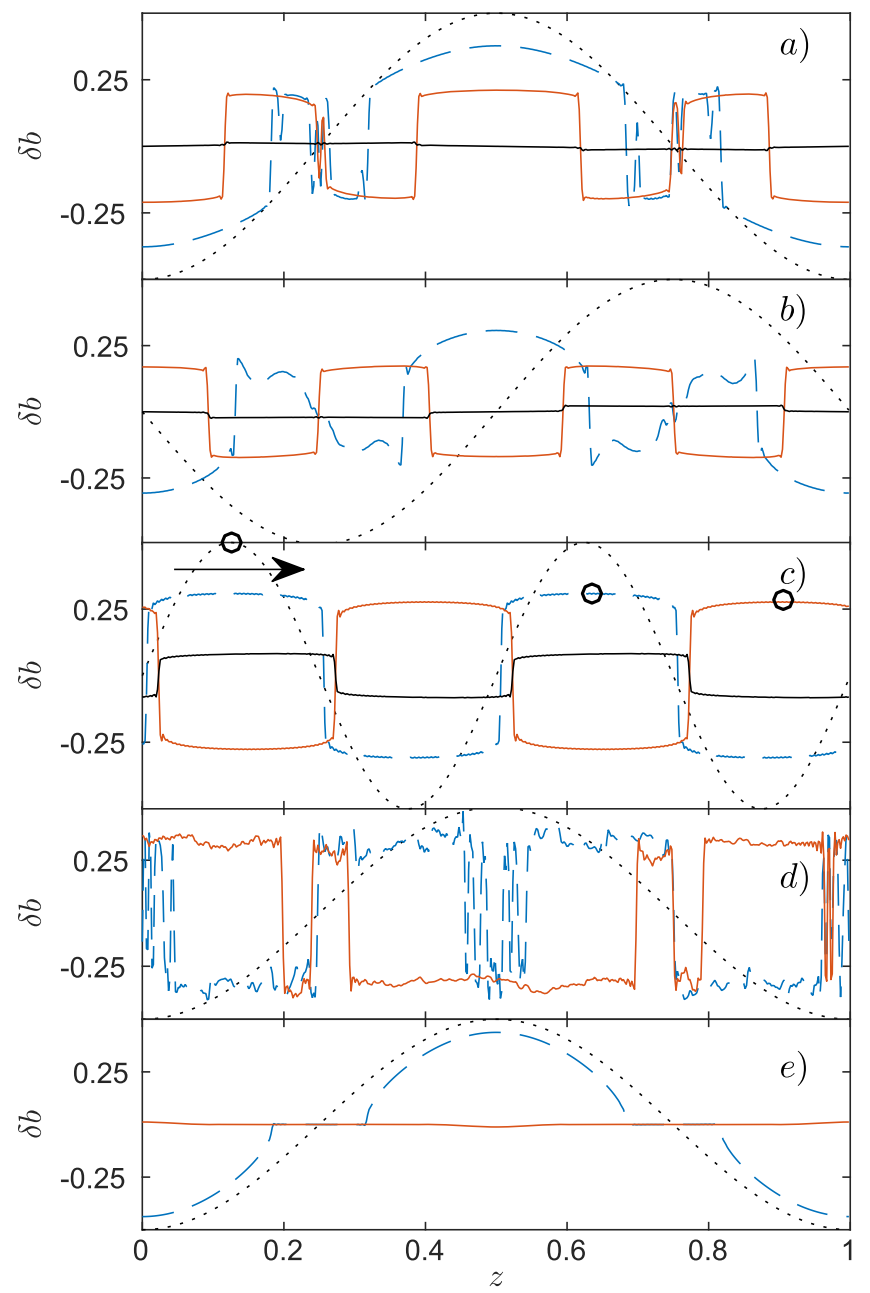

Figure 1. Evolution of $\delta b=B_{x} / B_{z 0}$ in a $\beta=100$ plasma. Panels (a)-(c) show solutions of the full collisionless LF Equations (2)-(6) in one dimension, starting from (a) $\delta b(0)=-0.5 \cos (2 \pi z)$, (b) $u_{x}(0)=-0.5 v_{A} \sin (2 \pi z)$ (linearly, this $u_{x}(0)$ leads to $\delta b=-0.5 \cos (2 \pi z)$ ), and (c) a traveling wave $\delta b(0)=-u_{x}(0) / v_{A}=0.5 \sin (4 \pi z)$. Panels (d) and (e) show standing-wave solutions of the nonlinear wave Equation (10), with (d) the collisionless closure (8), and (e) the Braginskii closure (9) (with $\omega_{A} \beta / \nu_{c}=100$ ). Each solution uses 512 Fourier modes. The figures show $\delta b$ at $t=0$ (black dotted line; $u_{x}(0)$ is shown in (b)), $\delta b$ at $t=\tau_{A} / 2$ (blue dashed line; $t=3 \tau_{A} / 2$ in (c)), $\delta b$ at $t=3 \tau_{A}$ (red solid line), and $u_{x} / v_{A}$ at $t=3 \tau_{A}$ (black solid line; only in (a)-(c)), where $\tau_{A}=2 \pi / \omega_{A}$. The circles in (c) show the same position on the wave as it evolves, illustrating its decrease in speed as the wave damps. Note the strong damping of velocity at late times in (a)-(c) (the wave is not fully interrupted by the final time shown in (c)), and the decay of the perturbation to $\delta b<\left(\delta b_{0}\right)_{\max }$ by $t=3 \tau_{A}$ in (e). The highly nonlinear behavior in each case shown here starkly contrasts with the almost perfectly linear evolution of an MHD SA fluctuation at these parameters.

of Equation (10) is $\sim \partial_{z}^{2}\left(\delta b^{3}\right)$, which lowers maxima of $\delta b^{2}$ while increasing minima, leading to constant- $B$ steps. With the Braginskii closure (Figure 1(e)), in contrast to the collisionless case, regions of small $\delta b$ have smaller $|\Delta|$ and thus decrease to zero before $\Delta=-2 / \beta$. Further, since the nonlinearity is diffusive, the field decays (over the timescale $\left.\tau_{\text {decay }} \sim \beta \delta b(0)^{2} / \nu_{c}\right)$, leaving ${ }^{7}$ small $\delta b<(\delta b)_{\max }$ fluctuations.

\footnotetext{
7 This estimate for $\tau_{\text {decay }}$ can be derived by setting $\Delta$ (Equation (9)) equal to the firehose limit in Equation (10) and solving the resulting differential equation. It is well matched by numerical solutions.
} 


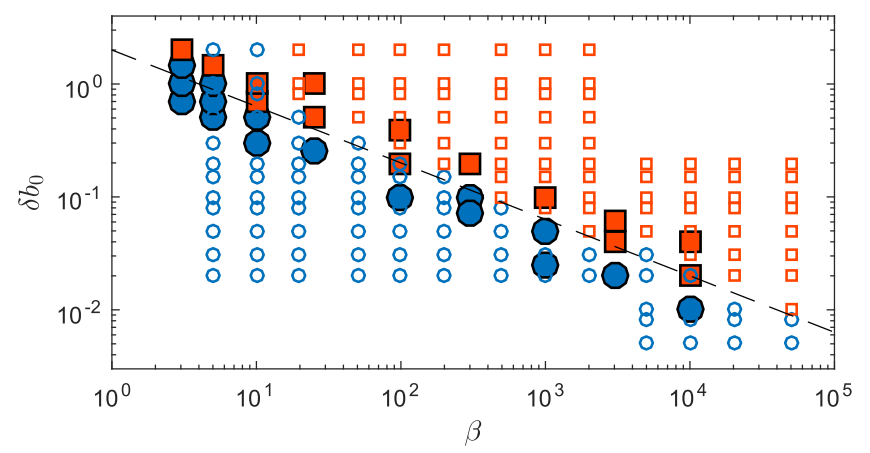

Figure 2. Numerical confirmation of the scaling (11). A red square indicates that an initial magnetic perturbation was interrupted before a half cycle (as in Figure 1), while a blue circle indicates that the perturbation flipped polarity without interruption. Large filled symbols show results from the LF Equations (2)-(6), while small hollow symbols show solutions of Equation (10) with the collisionless closure (8). The dashed line is $\delta b_{0}=2 \beta^{-1 / 2}$.

Figure 2 confirms the predictions of Equation (11), illustrating essentially perfect agreement for $\beta \gtrsim 10$. At $\beta \lesssim 10$ largeamplitude waves are still interrupted in the LF model, although solutions of Equation $(10)$ (which required $\delta b \ll 1$ ) deviate from Equation (11). We have also confirmed the scaling (12) for Braginskii MHD (not shown).

So far, we have considered only $1 \mathrm{D}$ evolution within the LF model-what caveats should be applied for more realistic conditions? The reader may wonder about the imposition of a mirror (but no firehose) limit in Figures 1(b)-(c). This is required because our model cannot capture the mirror instability, which gives rise to growing modes at $k_{\perp} \gg k_{\|}$. However, kinetic results (Kunz et al. 2014; Hellinger \& Trávníček 2015; Rincon et al. 2015; Melville et al. 2016) show that mirror fluctuations limit $\Delta$ by trapping particles, allowing $B$ to continue increasing while maintaining $\Delta=1 / \beta$. Further, the temporal growth of the mirror instability $\left|\delta B_{\|} / B_{0}\right| \sim(|\nabla \boldsymbol{u}| t)^{2 / 3}$ (Rincon et al. 2015) is slow enough that mirrors generated by an SA wave will not saturate and significantly scatter particles if $u_{x}(0) / v_{A}<1$. Thus, following a $\boldsymbol{u}$ perturbation with $B^{-1} d B / d t>0$, mirrors grow to limit $\Delta=1 / \beta$; then, once $B^{-1} d B / d t<0, \Delta$ immediately starts decreasing, while the (small) mirror fluctuations decay at the rate $\gamma \sim \Omega_{c} / \beta$ (Melville et al. 2016). This implies that SA waves cannot circumvent the limit (11) by starting from $B=0$ or $\Delta>0$ (see Figure 1(b)). Oblique firehose fluctuations (Yoon et al. 1993; Hellinger \& Trávníček 2008) are also not captured by our model, and these may change the nonlinear behavior by scattering particles (Kunz et al. 2014), potentially disrupting the angular field structure. ${ }^{8}$ Again, however, they cannot circumvent the amplitude limit itself; they become active only once $\Delta<-2 / \beta$, when the wave restoring force has already disappeared. We thus stress that, although the nonlinear outcome of wave interruptions (Figure 1) may be modified by the addition of other kinetic physics, our basic result-that collisionless SA fluctuations cannot exist in their linear wave form above the limit (11) — is robust. Its derivation is not sensitive to details of heat fluxes or particle scattering at the microinstability boundaries, relying purely on the physics

\footnotetext{
8 The angular magnetic structures themselves may also scatter particles, with $\nu_{c} \sim k_{\|} c_{s}$. This could cause faster damping of a wave once it hits the interruption limit and becomes square (or perhaps earlier for traveling waves, which can become square before $\Delta=-2 / \beta$; see Figure 1(c)).
}

of pressure-anisotropy generation due to magnetic moment conservation.

\section{IMPLICATIONS}

Given the ubiquity of Alfvén waves in space and astrophysical plasmas, the implications of the stringent constraint (1) on their amplitude at high $\beta$ may be dramatic, with applications ranging from the intracluster medium (Zhuravleva et al. 2014), to hot (collisionless) accretion disks (Quataert \& Gruzinov 1999) and the solar wind near Earth (Bruno \& Carbone 2013). We leave much of the discussion of these applications to future work, briefly considering possible observational evidence for the effect in the solar wind and the implications for magnetized turbulence (Goldreich \& Sridhar 1995). Note that, in contrast to results presented here, linear damping of long-wavelength, low-frequency SA waves at high $\beta$ is negligible if $\Omega_{c} \beta^{-1} / \omega_{A} \gg 1$ (Foote \& Kulsrud 1979; Achterberg 1981; Cramer 2011).

Alfvén waves are fundamental to solar wind physics, and our results are most relevant to regions where $\beta \gtrsim 1$, at solar radii 1 au (Mullan \& Smith 2006; Bruno \& Carbone 2013). Specifically, propagation of large-amplitude SA waves into a $\beta \gtrsim 1$ plasma may naturally form rotational field-line discontinuities (Borovsky 2008; Miao et al. 2011), heating the plasma as the wave interrupts. An interesting observational feature that may be related to this is the appearance of a distinct, magnetically dominated, population of fluctuations at increasing solar radii (Tu \& Marsch 1991; Bruno et al. 2007; Bruno \& Carbone 2013). This population's sudden appearance across a range of latitudes (Bavassano et al. 1998) suggests it does not arise through continuous evolution of turbulence (see Figure 2 of Bruno et al. 2007). Such characteristics would be expected from SA wave interruption in regions where $\beta \gtrsim 1$, a scenario that is also consistent with the observed excess of magnetic energy (Goldstein et al. 1995; Roberts 2010; Chen et al. 2013; Oughton et al. 2015). A prediction of our scenario is a correlation between $\beta$ and regions with magnetically dominated, rotationally discontinuous, structures.

The implications of our results for magnetized turbulence in collisionless plasmas are potentially dramatic. A striking conclusion, which holds independently of the details of interrupted structures, is that perturbations in a collisionless plasma with energy densities on the order of $B_{0}^{2}$ (i.e., $|\boldsymbol{u}| \sim v_{A}$ ) are immediately damped-that is, the plasma behaves as a fluid with Reynolds number $\lesssim 1$. Where does this perturbation energy go? Because of the same energy transfer term responsible for damping traveling waves, $\partial_{t} E_{\mathrm{th}} \sim \int d \boldsymbol{x} \Delta p B^{-1} d B / d t$, if $\Delta<0$, a decreasing field directly transfers large-scale kinetic energy into plasma heating (Sharma et al. 2006). A turbulent cascade is thus no longer necessary for collisionless plasmas to absorb the energy input by a continuous mechanical forcing (Kunz et al. 2010), and it is unclear if any of the energy provided on large scales cascades to smaller scales as traditionally assumed. However, such physics is well beyond the scope of this work and we conclude here by simply reiterating that the immediate disruption of SA fluctuations when $\delta B_{\perp} / B_{0} \gtrsim \beta^{-1 / 2}$ severely limits the application of standard magnetized turbulence phenomenologies (Goldreich \& Sridhar 1995) to high- $\beta$ collisionless plasmas.

A variety of fundamental questions about the nonlinear interruption of shear-Alfvén waves remain for future studies, particularly concerning higher-dimensional microinstabilities 
(e.g., oblique firehose). Fully kinetic simulations will be key to understanding this physics better. Given the robustness and generality of our result, its appearance in a variety of models, and the stringent nature of the $\delta B_{\perp} / B_{0} \lesssim \beta^{-1 / 2}$ condition, we anticipate a range of future applications to heliospheric, astrophysical, and possibly laboratory (Forest et al. 2015; Gekelman et al. 2016) plasmas.

It is a pleasure to thank S. Bale, C. H. K. Chen, S. Cowley, M. Kunz, and M. Strumik for useful discussions. This work was supported in part by the Sherman Fairchild foundation (J.S.), the Gordon and Betty Moore Foundation to Lars Bildsten, Eliot Quataert and E. Sterl Phinney, Simons Investigator awards from the Simons Foundation (E.Q.), NSF grant AST 13-33612 (E.Q.), and by UK STFC and EPSRC grants (A.A.S.).

\section{REFERENCES}

Achterberg, A. 1981, A\&A, 98, 161

Alfvén, H. 1942, Natur, 150, 405

Bavassano, B., Pietropaolo, E., \& Bruno, R. 1998, JGR, 103, 6521

Borovsky, J. E. 2008, JGRA, 113, A08110

Braginskii, S. I. 1965, RvPP, 1, 205

Bruno, R., \& Carbone, V. 2013, LRSP, 10, 2

Bruno, R., D’Amicis, R., Bavassano, B., Carbone, V., \& Sorriso-Valvo, L. 2007, AnGeo, 25, 1913

Chandrasekhar, S., Kaufman, A. N., \& Watson, K. M. 1958, RSPSA, 245, 435

Chen, C. H. K., Bale, S. D., Salem, C. S., \& Maruca, B. A. 2013, ApJ, 770, 125

Cramer, N. F. 2011, The Physics of Alfvén Waves (New York: Wiley)

Del Zanna, L., Velli, M., \& Londrillo, P. 2001, A\&A, 367, 705

Foote, E. A., \& Kulsrud, R. M. 1979, ApJ, 233, 302

Forest, C. B., Flanagan, K., Brookhart, M., et al. 2015, JPIPh, 81, 345810501

Gekelman, W., Pribyl, P., Lucky, Z., et al. 2016, RScI, 87, 025105

Goldreich, P., \& Sridhar, S. 1995, ApJ, 438, 763
Goldstein, B. E., Smith, E. J., Balogh, A., et al. 1995, GeoRL, 22, 3393

Hamabata, H. 1993, ApJ, 406, 563

Heidbrink, W. W. 2008, PhPl, 15, 055501

Hellinger, P., \& Trávníček, P. M. 2008, JGRA, 113, A10109

Hellinger, P., \& Trávníček, P. M. 2015, JPIPh, 81, 305810103

Kulsrud, R. M. 1983, in Handbook of Plasma Physics, ed. R. N. Sagdeev \& M. N. Rosenbluth (Princeton, NJ: Princeton Univ. Press), 115

Kunz, M. W., Schekochihin, A. A., Cowley, S. C., Binney, J. J., \& Sanders, J. S. 2010, MNRAS, 410, 2446

Kunz, M. W., Schekochihin, A. A., \& Stone, J. M. 2014, PhRvL, 112, 205003

Marsch, E. 2006, LRSP, 3, 1

Medvedev, M. V., Shevchenko, V. I., Diamond, P. H., \& Galinsky, V. L. 1997, $\mathrm{PhPl}, 4,1257$

Melville, S., Schekochihin, A. A., \& Kunz, M. W. 2016, MNRAS, 459, 2701

Miao, B., Peng, B., \& Li, G. 2011, AnGeo, 29, 237

Mullan, D. J., \& Smith, C. W. 2006, SoPh, 234, 325

Ng, C. S., \& Bhattacharjee, A. 1996, ApJ, 465, 845

Ofman, L. 2010, LRSP, 7, 4

Oughton, S., Matthaeus, W. H., Wan, M., \& Osman, K. T. 2015, RSPTA, 373, 20140152

Parker, E. N. 1958, PhRv, 109, 1874

Quataert, E., \& Gruzinov, A. 1999, ApJ, 520, 248

Rincon, F., Califano, F., Schekochihin, A. A., \& Valentini, F. 2016, PNAS, 113,3950

Rincon, F., Schekochihin, A. A., \& Cowley, S. C. 2015, MNRAS, 447, L45

Roberts, D. A. 2010, JGRA, 115, A12101

Rosenbluth, M. N. 1956, Los Alamos Sci. Lab. Rep. LA-2030

Santos-Lima, R., de Gouveia Dal Pino, E. M., Kowal, G., et al. 2014, ApJ, 781,84

Schekochihin, A. A., Cowley, S. C., \& Dorland, W. 2007, PPCF, 49, A195

Schekochihin, A. A., Cowley, S. C., Rincon, F., \& Rosin, M. S. 2010, MNRAS, 405, 291

Sharma, P., Hammett, G. W., Quataert, E., \& Stone, J. M. 2006, ApJ, 637, 952

Snyder, P. B., Hammett, G. W., \& Dorland, W. 1997, PhPl, 4, 3974

Tu, C.-Y., \& Marsch, E. 1991, AnGeo, 9, 319

Velli, M. 1993, A\&A, 270, 304

Yoon, P. H., Wu, C. S., \& de Assis, A. S. 1993, PhFlB, 5, 1971

Zhuravleva, I., Churazov, E., Schekochihin, A. A., et al. 2014, Natur, 515, 85 